\title{
Systemic Treatment in Advanced Phyllodes Tumor of the Breast: A Multi-Institutional European Retrospective Case-Series Analyses
}

Elena Palassini ( $\nabla$ elena.palassini@istitutotumori.mi.it)

Fondazione IRCCS Istituto Nazionale dei Tumori https://orcid.org/0000-0003-2257-4681

\section{Olivier Mir}

Gustave Roussy

\section{Giovanni Grignani}

Istituto per la Ricerca e la Cura del Cancro di Candiolo: Candiolo Cancer Institute

\section{Bruno Vincenzi}

Campus Bio-Medico University: Universita Campus Bio-Medico di Roma

\section{Hans Gelderblom}

Leids Universitair Medisch Centrum

\section{Ana Sebio}

Hospital de Sant Pau: Hospital de la Santa Creu i Sant Pau

\section{Claudia Valverde}

Vall d'Hebron Hospital: Vall d'Hebron Hospital Universitari

\section{Giacomo Giulio Baldi}

Ospedale di Prato Santo Stefano

\section{Antonella Brunello}

IOV IRCCS: Istituto Oncologico Veneto Istituto di Ricovero e Cura a Carattere Scientifico

\section{Giovanni Gerardo Cardellino}

Santa Maria della Misericordia University Hospital: Presidio Ospedaliero Universitario Santa Maria della Misericordia

\section{Andrea Marrari}

Humanitas Clinical and Research Center IRCCS

\section{Giuseppe Badalamenti}

University of Palermo: Universita degli Studi di Palermo

\section{Javier Martin-Broto}

Universidad de Sevilla: Universidad de Sevilla

\section{Virginia Ferraresi}

Regina Elena Institute Institute for Hospitalization and Care Scientific: Istituto Regina Elena

\section{Michela Libertini}

Fondazione Poliambulanza Istituto Ospedaliero 


\section{Salvatore Turano}

Azienda Ospedaliera di Cosenza

\section{Ithar Gataa}

Gustave Roussy

\section{Paola Collini}

Fondazione IRCCS Istituto Nazionale dei Tumori

\section{Angelo Paolo Dei Tos}

University of Padova: Universita degli Studi di Padova

\section{Massimiliano Gennaro}

Fondazione IRCCS Istituto Nazionale dei Tumori

\section{Federica Bini}

Fondazione IRCCS Istituto Nazionale dei Tumori

\section{Salvatore Provenzano}

Fondazione IRCCS Istituto Nazionale dei Tumori

\section{Salvatore Lo Vullo}

Fondazione IRCCS Istituto Nazionale dei Tumori

\section{Luigi Mariani}

Fondazione IRCCS Istituto Nazionale dei Tumori

\section{Axel Le Cesne}

Gustave Roussy

\section{Paolo Giovanni Casali}

Fondazione IRCCS Istituto Nazionale dei Tumori

\section{Research Article}

Keywords: Breast Tumor, Phyllodes, Sarcoma, Chemotherapy, Advanced Setting.

Posted Date: October 29th, 2021

DOl: https://doi.org/10.21203/rs.3.rs-919256/v2

License: (c) (i) This work is licensed under a Creative Commons Attribution 4.0 International License. Read Full License

Version of Record: A version of this preprint was published at Breast Cancer Research and Treatment on February 12th, 2022. See the published version at https://doi.org/10.1007/s10549-022-06524-4. 


\section{Abstract}

Background: We aimed at investigating outcome of systemic treatments in advanced breast PT.

Methods: All cases of advanced breast PT treated with systemic treatments from 1999 to 2019, in one of the referral sarcoma centres involved in the study, were retrospectively reviewed.

Results: 56 female patients were identified. Median age was 52 (range 25-76) years. Patients re-ceived a median number of 2 systemic treatments (range 1-4). Best responses according to RECIST were: 1 (3.7\%) CR, 11 (40.7\%) PR, 6 (22.2\%) SD, 9 (33.3\%) PD with anthracyclines plus ifosfamide (Al); 2 (16.7\%) PR, 4 (33.3\%) SD, 6 (50.0\%) PD with anthracycline alone; 3 (18.8\%) PR, 4 (25.0\%) SD, 9 (56.3\%) PD with highdose ifosfamide given as a continuous infusion (HD-IFX); 3 (20.0\%) SD, 12 (80.0\%) PD with a gemcitabine-based regimen (with 2 patients not evaluable); 1 (8.3\%) PR, 2 (16.7\%) SD, 9 (75.0\%) PD with trabectedin (with 1 patient not evaluable); 1 (16.7\%) PR, 1 (16.7\%) SD, 4 (66.7\%) PD with tyrosine-kinase inhibitors (TKI). The median PFS were: 5.7 (IQR, 2.5-9.1) months with Al; 3.2 (IQR, 2.2-5.0) months with anthracycline alone; 3.4 (IQR, 1.4-6.7) months with HD-IFX; 2.1 (IQR, 1.4-5.2) months with gemcitabinebased chemotherapy; 1.8 (IQR, 0.7-6.6) months with trabectedin; 3.4 (IQR, 3.1-3.8) months with TKI. With a median follow-up of 35.3 (IQR, 17.6-66.9) months, OS from the start of first-line systemic treatment was 15.2 (IQR, 7.6-39.6) months.

Conclusion: In this series of advanced PT (to our knowledge, the largest reported so far), Al was associated with a high rate of responses, however with a median PFS of 5.7 months. Other systemic treatments were poorly active.

\section{Background}

Accounting for less than $1 \%$ of all breast neoplasms, phyllodes tumors of the breast are a rare group of biphasic fibroepithelial neoplasms with a double-layered epithelial component surrounded by a hypercellular stromal/mesenchymal component. According to WHO, they are classified into benign, borderline or malignant on the basis of a combination of several pathological features, including the degree of stromal hyper-cellularity, mitosis, cytological atypia, stromal overgrowth and nature of tumor borders/margins. Most phyllodes tumors are benign, while malignant lesions account for $10-20 \%$ of all cases [1].

Overall, phyllodes tumors have a low risk of developing metastases (about 2\%), reaching $20-25 \%$ if they are malignant [2-4]. The management of metastatic disease is poorly studied and it is generally based on the use of soft tissue sarcoma systemic treatments. However, given the rarity of this disease and its low metastatic potential, available data on systemic treatments are limited and our knowledge is based on small case reports or individual case reports [5-8].

The aim of this international, collaborative study, including several referral sarcoma centers in Europe, was to report on activity and efficacy of systemic treatments in adult patients with advanced PT. 


\section{Materials And Methods}

All patients with locally advanced/metastatic PT of breast treated with systemic treatment, over the period from 1999 to 2019, in one of the centers involved in the study, were included in this analysis. Patients treated within the context of the Italian Rare Cancer Network were also considered. Patients treated in adjuvant/neoadjuvant setting were excluded.

Data were extracted from clinical databases. The following data were collected: date of birth, gender, date of diagnosis, treatments received for local disease, treatments received for advanced disease, survival. For each systemic treatment received for advanced disease date of start, toxicity related to treatment, radiological response and time to progression were reported. Details on pathological characteristics of tumor at diagnosis were also recorded.

Toxicity was recorded using National Cancer Institute Common Toxicity Criteria (CTCAE v5.0) [9]. Since the analysis was focused on activity and efficacy of systemic treatments, only grade 3 or 4 adverse events were sought.

Radiological response was locally assessed according to Response Evaluation Criteria in Solid Tumors (RECIST, version 1.1) [10].

Descriptive statistics and frequency tabulation were used to summarize patient and tumor characteristics. Overall response rate (ORR) was computed as the frequency of patients with complete or partial tumor response among the patients evaluable for response. The corresponding confidence intervals are two-sided $95 \%$ exact intervals, calculated by use of the binomial formula.

Progression-free survival (PFS) and overall survival (OS) were estimated with Kaplan-Meier method [10]. For PFS, the event time was computed from the date of treatment start to the date of progression or death or last follow-up. Patients undergoing surgical treatment after systemic treatment were censored at the time of progressive disease after surgical resection or at the last follow-up. For OS, the event time was computed from the date of treatment start to the date of death due to any cause or last follow-up. Eventfree patients were censored at the date of last patient contact. Statis-tical analyses were carried out with SAS (version 9.4, SAS Institute, Cary, NC, USA) and R software (version 4.0.2, R Foundation for Statistical Computing, Vienna, Austria).

Approval by the institutional review board of each institution was obtained. Written informed consent was obtained as required by local regulation.

\section{Results}

Fifty-six patients with advanced PT treated with systemic treatments over the study period were identified, 2 (4\%) with locally advanced disease and 54 (96\%) with metastatic disease at the start of the first systemic treatment. All patients were female. Median age at time of the first systemic treatment was 52 
years (interquartile range, IQR: $42-57$ years). Initial diagnosis was malignant PT in 50 (89.3\%) patients and border-line PT in 5 (8.9\%) patients (1 missing data). Patients and tumor characteristics are detailed in Table 1.

Table 1. Patients and tumor characteristics.

\begin{tabular}{|c|c|c|}
\hline & Number & $\%$ \\
\hline Number of Patients & 56 & 100 \\
\hline \multicolumn{3}{|l|}{ Age } \\
\hline Median, y & 52 & \\
\hline IQ Range, y & $42-57$ & \\
\hline \multicolumn{3}{|l|}{ Sex } \\
\hline Female & 56 & 100 \\
\hline \multicolumn{3}{|l|}{ Diagnosis } \\
\hline Borderline PT & 50 & 89.3 \\
\hline Malignant PT & 5 & 8.9 \\
\hline Missing & 1 & 1.8 \\
\hline \multicolumn{3}{|c|}{ Extension at systemic treatment start } \\
\hline Locally advanced & 2 & 3.6 \\
\hline Metastatic & 54 & 96.4 \\
\hline
\end{tabular}

A median number of systemic treatment lines of 2 was administered. A total number of one, two, three or more than three lines were administered in 21 (37.5\%), 20 (35.7\%), 10 (17.9\%) and $5(8.9 \%)$ patients, respectively. The most used systemic treatment regimens were: anthracycline plus ifosfamide ( 27 patients, $48.2 \%$ ), anthracycline as single agent (12 patients, $21.4 \%$ ), high-dose ifosfamide given as a continuous infusion for 14 days (16 patients, $28.6 \%$ ), gemcitabine-based regimens (17 patients, $30.4 \%$ ), trabectedin (13 patients, 23.2\%) tyrosine-kinase inhibitors (6 patients, 10.7\%). The sequence of systemic treatments received by each patient is listed in Table 2. Details on activity, efficacy and toxicity of each of these systemic treatments are described hereafter. After systemic treatment, seventeen patients $(30.4 \%)$ received also surgery for metastatic disease.

The median OS from the start of the first systemic treatment was 15.2 months (IQR, 7.6-39.6 months). At the time of the analyses, with a median follow-up of 35.3 months (IQR, 17.6-66.9 months) from the start of the first systemic treatment, 38 patients had died. 18 patients were alive: 12 with disease and 6 with no 
evidence of disease, after one line of systemic treatment and a subsequent surgery. Figure 1 shows the OS curve of all patients included in the analyses.

Table 2. Sequences of systemic treatments received by each patient. 


\begin{tabular}{|lll|}
\hline & Number & $\%$ \\
\hline A & 4 & 7.1 \\
\hline A+HDIFX & 2 & 3.6 \\
\hline A+G & 1 & 1.8 \\
\hline A+O & 3 & 5.4 \\
\hline Al & 10 & 17.9 \\
\hline Al+HDIFX & 3 & 5.4 \\
\hline Al+HDIFX+G & 1 & 1.8 \\
\hline Al+HDIFX+G+TKI & 1 & 1.8 \\
\hline Al+HDIFX+TKI & 2 & 3.6 \\
\hline Al+HDIFX+TKI+G & 1 & 1.8 \\
\hline Al+G & 1 & 1.8 \\
\hline Al+G+HDIFX & 1 & 1.8 \\
\hline Al+G+HDIFX+A & 1 & 1.8 \\
\hline Al+G+O+T & 1 & 1.8 \\
\hline Al+TKI & 1 & 1.8 \\
\hline Al+O & 2 & 3.6 \\
\hline Al+O+G+O & 1 & 1.8 \\
\hline Al+T+TKI & 1 & 1.8 \\
\hline HDIFX+A & 1 & 1.8 \\
\hline HDIFX+G & 1 & 1.8 \\
\hline G & 1 & 1.8 \\
\hline G+HDIFX & 1 & 1.8 \\
\hline G+T & 1 & 1.8 \\
\hline G+T+HDIFX & 1 & 1.8 \\
\hline G+T+TKI & 1 & \\
\hline O+TKI+O & 1 & 1.8 \\
\hline
\end{tabular}




\begin{tabular}{|lll|}
\hline $\mathbf{O}+\mathbf{O}$ & 1 & 1.8 \\
\hline $\mathbf{O + T K I}$ & 1 & 1.8 \\
\hline $\mathbf{O + T K I + O}$ & 1 & 1.8 \\
\hline $\mathbf{T}$ & 2 & 3.6 \\
\hline $\mathbf{T + G}$ & 1 & 1.8 \\
\hline Overall & 56 & 100 \\
\hline
\end{tabular}

${ }^{1}$ Abbreviation: Al: anthracycline plus ifosfamide; A: anthracycline as single agent; HDIFX: high-dose ifosfamide; G: gemcitabine-based regimen; T: trabectedin; TKI: tyrosine-kinase inhibitors; O: other treatments

\subsection{Anthracycline plus ifosfamide.}

Twenty-seven patients received anthracycline plus ifosfamide (Al), all as first-line systemic treatment. Epirubicin plus ifosfamide was used in 19 (70.4\%) patients, doxorubicin plus ifosfamide in 7 (25.9\%) patients and liposomal doxorubicin plus ifosfamide in 1 (3.7\%) patient. Out of 19 patients evaluable for toxicity, grade 3-4 toxicity events were observed in 14 (73.7\%) patients. Grade 3-4 toxicities events included: anemia, neutropenia, thrombocytopenia, febrile neutropenia and mucositis.

All 27 patients were evaluable for response. Best response according to RECIST was: complete response (CR) in $1(3.7 \%)$ patient, partial response (PR) in 11 (40.7\%) patients, stable disease (SD) in $6(22.2 \%)$ patients and progressive disease (PD) in 9 (33.3\%) patients. The ORR was $44.4 \%$ (95\% Cl: $25.5 \%-64.7 \%)$.

The median PFS was 5.7 months (IQR, 2.5-9.1 months). Among the 18 patients who obtained CR, PR or SD, 7 (38.9\%) patients and 5 patients (27.8\%) were free from progression after 9 months and one year, respectively, from the start of Al. Figure $2 \mathrm{~A}$ shows the PFS curve of patients who received Al.

\subsection{Anthracycline as single agent.}

Twelve patients received anthracycline as single agent: as first-line systemic treatment in 10 (83.3\%) cases, as second-line in $1(8.3 \%)$ case and as further line in $1(8.3 \%)$ additional case. Doxorubicin was used in $11(91.7 \%)$ patients and epirubicin in $1(8.3 \%)$ patient. Out of 10 patients evaluable for toxicity, grade 3-4 toxicity events were observed in $4(40 \%)$ patients. Grade 3-4 toxicities events included: anemia, neutropenia, febrile neutropenia and thrombosis.

All 12 patients were evaluable for response. Best response according to RECIST was: PR in 2 (16.7\%) patients, SD in $4(33.3 \%)$ patients and PD in $6(50.0 \%)$ patients. The ORR was $16.7 \%$ (95\% Cl: $2.1 \%-48.4 \%)$.

The median PFS was 3.2 months (IQR, 2.2-5.0 months). Among the 6 patients who obtained PR or SD, 2 $(33.3 \%)$ patients were free from progression after six months from the start of anthracycline 
monotherapy. Figure 2B shows the PFS curve of patients who received anthracycline as single agent.

\subsection{High-dose ifosfamide}

Sixteen patients received high-dose ifosfamide (HD-IFX) given as a continuous infusion for 14 days, as first-line systemic treatment in $2(12.5 \%)$ cases, as second-line in $11(68.8 \%)$ cases, as third line in 3 $(18.8 \%)$ cases. Ten patients had been previously treated with Al. Out of 13 patients evaluable for toxicity, grade 3-4 toxicity events were observed in 3 patients (23.1\%). Grade 3-4 toxicities events included: anemia, vomiting and asthenia.

All patients were evaluable for response. Best response according to RECIST was: PR in $3(18.8 \%)$ patients, SD in 4 (25.0\%) patients and PD in 9 (56.3\%) patients. The ORR was $18.8 \%$ (95\% Cl: 4.1\%-45.7\%). Among the 3 patients obtaining PR as best response, 2 patients had already received Al, with PD and PR, respectively.

The median PFS was 3.4 months (IQR, 1.4-6.7 months). Among the 7 patients who obtained PR or SD, 2 $(28 \%)$ patients were free from progression after one year from the start of HD-IFX. Figure $2 \mathrm{C}$ shows the PFS curve of patients who received HD-IFX.

\subsection{Gemcitabine-based regimens.}

Seventeen patients received a gemcitabine-based regimen, as first-line systemic treatment in $5(29.4 \%)$ cases, as second-line in $7(41.2 \%)$ cases, as third-line in 4 (23.5\%) cases and as further-line in 1 (5.9\%) case. Gemcitabine plus docetaxel was used in $8(47.1 \%)$ patients, gemcitabine plus dacarbazine in 2 $(11.8 \%)$ patients and gemcitabine as single agent in $7(41.2 \%)$ patients. Out of 14 patients evaluable for toxicity, grade 3-4 toxicity events were observed in 6 patients (42.9\%). Grade 3-4 toxicities events included: anemia, neutropenia, thrombocytopenia and asthenia.

Fifteen patients were evaluable for response. Best response according to RECIST was: SD in 3 patients (20.0\%) and PD in 12 patients (80.0\%). No objective responses were observed.

The median PFS was 2.1 months (IQR, 1.4-5.2 months). In the group of 3 patients who obtained SD, one (33.3\%) patient was free from progression after one year from the start of gemcitabine-based regimen. Figure 2D shows the PFS curve of patients who received a gemcitabine-based regimen.

\subsection{Trabectedin.}

Thirteen patients received trabectedin, as first-line systemic treatment in $3(23.1 \%)$ cases, as second-line in $6(46.2 \%)$ cases, as third line in $3(23.1 \%)$ cases and as further line in one (7.7\%) patient. Out of 12 patients evaluable for toxicity, grade 3-4 toxicity events were observed in 1 patient (8.3\%). Grade 3-4 toxicities events were restricted to liver toxicity.

Twelve patients were evaluable for response. Best response according to RECIST was: PR in 1 (8.3\%) patient, SD in 2 (16.7\%) patients and PD in 9 patients (75.0\%). The ORR was 8.3\% (95\% Cl: 0.2\%-38.5\%). 
The median PFS was 1.8 months (IQR, 0.7-6.6 months). In the group of 3 patients who obtained PR or SD, $2(66.7 \%)$ patients were free of progression after one year from the start of trabectedin. Figure 2E shows the PFS curve of patients who received trabectedin.

\subsection{Tyrosine-kinase inhibitors}

Six patients received tyrosine-kinase inhibitors (TKI), as second-line systemic treatment in $3(50.0 \%)$ cases, as third line in $2(33.3 \%)$ cases, as further-line in 1 (16.7\%) case. Pazopanib was used in $5(83.3 \%)$ patients and regorafenib in $1(16.7 \%)$ patient. All 6 patients were evaluable for toxicity; grade 3-4 toxicity events were observed in 2 (33.3\%) patients. Grade 3-4 toxicities events included: diarrhea and dental abscess.

Six patients were evaluable for response. Best response according to RECIST was: PR in $1(16.7 \%)$ patient (receiving regorafenib), SD in 1 (16.7\%) patient and PD in 4 (66.7\%) patients. The ORR was $16.7 \%$ (95\% Cl: 0.4\%-64.1\%).

The median PFS was 3.4 months (IQR, 3.1-3.8 months). All 2 patients who obtained PR or SD progressed before six months from the start of TKI. Figure 2F shows the PFS curve of patients who received TKI.

\section{Discussion}

In this international retrospective observational study involving 15 European cancer centers, 56 patients with advanced PT treated with systemic therapy were included. The combination of anthracyclines plus ifosfamide provided a tumor response in almost $45 \%$ of patients, with a median PFS of almost 6 months and a few patients being progression-free at 2-3 years. This is consistent with what is seen with the same regimen in soft tissue sarcomas. A low response rate was seen to other systemic treatments.

With all the limitations of a retrospective analysis, this is the largest series on outcome of several systemic treatments in patients with advanced PT. Given the rarity of this disease and the relative low incidence of metastases, available data on systemic treatments in this disease are scarce. Mitus et al. in 2016 described the impact of systemic treatment in 30 metastatic PT patients over a 50-year time interval [6]. Subsequently, Neron et al. published the experience of several French centers within the French Sarcoma Group (FSG-GETO) in the management of metastatic PT patients, including 37 patients treated with systemic treatment over the period 2000-2016 [5]. More recently, Parkers et al. reported the experience of MD Anderson Cancer Center, with 31 metastatic PT patients receiving systemic treatment over the period 1993-2015 [8].

In this series, the combination of anthracycline plus ifosfamide was associated with a response rate (almost $45 \%$ ) similar to what previously observed by other authors. Neron et al. reported a clinical benefit in 6 out of 13 patients (almost 50\%) treated with this combination and Mitus et al. described $1 \mathrm{CR}$ and 2 PR in 3 patients [5,6]. In the MD Anderson Cancer Center series, anthracycline plus ifosfamide was associated with a response rate of $56 \%$ (10 out of 18 patients) [8]. With a response rate in this range, the 
activity of anthracycline plus ifosfamide in PT looks similar to the activity of this regimen in STS in general [12]. However, the median PFS was 6 months. In the group of patients who had obtained a response or a stabilization with this treatment, almost one third (5 out $18 \mathrm{pts}, 28 \%$ ) was free from progression after one year from the start of the treatment. On the basis of these results, the use of this combination may be encouraged in advanced PT, especially in symptomatic patients and/or if an integration with surgery is foreseen.

Anthracycline as single agent provided a low response rate (17\%) in this series, with a median PFS of 3 months. Previously published papers reported similar results $[5,6]$. Neron et al. described a clinical benefit in 1 out of 11 patients treated with doxorubicin alone, while Mitus et al. observed a PR in 4 out of 6 patients treated with doxorubicin alone, but in all the 3 cases survival was shorter than 9 months $[5,6]$.

The response rate with other chemotherapy regimens included in the current analyses was low, being $19 \%, 0 \%$ and $8 \%$, with HD-IFX, with gemcitabine-based regimens and with trabectedin, respectively. Of interest, one PR with HD-IFX was seen in a patient who had previously progressed on conventional dose of ifosfamide, given in addition to epirubicin. The median PFS associated to these regimens was low (3.4, 2.1, 1.8 months with HD-IFX, with gemcitabine-based regimens and with trabectedin, respectively), although a small number of patients treated with these regimens obtained long-lasting responses/stabilizations. Mitus et al. reported a PR in 1 out of 4 patients treated with ifosfamide alone (25\%, 6-month survival) and Neron et al. described a clinical benefit in 3 out of 6 patients (50\%) who received an alkylating agent alone. Gemcitabine-based regimens were associated with a response rate of $33 \%$ (3 out of 9 patients) in the experience of MD Anderson Cancer Center, but PFS was shorter than 3 months [8]. Trabectedin was not included in previously published studies on PT, aside from one PT patient reported by Grignani et al. who received trabectedin plus olaparib achieving a PR, in the context of a phase I/II study investigating this association in STS [13]. The results of the current analysis showed a poor outcome with HD-IFX, gemcitabine-based regimens and trabectedin in this disease. However, a possible role of HD-IFX and trabectedin, at least in a subset of PT patients, cannot be excluded.

No responses were seen with pazopanib in the current series. Of course, it should be considered that the number of patients included in this group was very limited. One response was seen with regorafenib, even if the duration of disease control was poor. Of interest, Neron et al. reported 1 response with regorafenib, while pazopanib was not reported to be used in the context of the FSG retrospective series [5]. The activity of another TKI, sunitinib, was previously reported by other authors [14]. Further investigations are needed to better understand the role of TKI.

In this series, the median OS from the start of first systemic treatment was 15 months. At a median follow-up of 35 months, 18 patients were alive. Of them, 6 patients were alive with no evidence of disease, after a first-line systemic treatment and a sub-sequent surgery. Of course, this analysis was not aimed to capture the impact of surgery in metastatic PT. However, as in STS in general, surgery may be an option in a selected group of metastatic PT patients [15]. 
With regard to pathological characteristics at the time of the diagnosis, a diagnosis of borderline PT was made in $5(8.9 \%)$ cases who had a subsequent evidence of meta-static recurrences, suggesting a possible aggressive behavior even in the lack of an up-front diagnosis of malignant PT.

\section{Conclusion}

In conclusion, in this series of advanced breast PT treated with systemic treat-ments, we observed that systemic treatment as used in other soft tissue sarcomas are associated with a distinct number of responses, which on average have a low duration, and though a few patients being progression-free at 23 years.

We think that these data might provide a benchmark for future trials on agents in this disease. Moreover, the present study confirms the value of international, collabo-rative, retrospective studies among referall centres to answer relevant clinical questions in very rare neoplasms.

\section{Abbreviations}

Breast Phyllodes Tumor (PT); High-dose ifosfamide (HD-IFX); Tyrosine-kinase inhibitors (TKI); Cancer Institute Common Toxicity Criteria (CTCAE); Response Evaluation Criteria in Solid Tumors (RECIST); overall response rate (ORR); Progression-free survival (PFS); Overall survival (OS); Anthracycline plus ifosfamide (Al); Complete response (CR); Partial response (PR); Stable disease (SD); Progressive disease (PD).

\section{Declarations}

Authors' contributions: Conceptualization, E.P. and P.G.C.; methodology, E.P., P.G.C., L.M.; software, S.LV; validation, E.P. and P.G.C.; formal analysis, S.LV., and L.M.; investigation, all.; resources, all.; data curation, E.P., and S.LV.; writing original draft preparation, E.P., and P.G.C.; writing review and editing, all.; visualization, F.B., and E.P.; supervision, P.G.C.. All authors have read and agreed to the published version of the manuscript.

Funding: The authors received no financial support for the research, authorship, and/or publication of this article.

Conflict of interest: The following represents disclosure information provided by authors of this manuscript. All relationships are considered compensated unless otherwise noted. Relationships are selfheld unless noted.

EP and SP reports institutional financial interest in Advenchen, Amgen-Dompe, Bayer, Epizyme, Eli Lilly, Daiichi-Sankyo, GlaxoSmithKline, Karyopharm, Novartis, Pfizer, PharmaMar, and SpringWorks Therapeutics. 
OM reports Advisory Board for Bayer, Blueprint Medicines, MSD, Pfizer, Invited Speaker for BMS, Eli-Lilly, Roche, Servier and Institutional, Financial interest as Local PI for Bayer, Blueprint Medicines, Eli-Lilly, Epizyme.

AS reports advisory role for Pharmamar and also travel and congress assistance, research, teaching from Pharmamar, teaching, travel and congress assistance from Lilly, Eisai and travel and congress assistance from Merc, Amgen, Sanofi.

CV reports Advisory Board for Bayer, Boehringer Ingelheim, GSK, Lilly, Mundipharma, Phar-maMar; Invited Speaker from Roche; Institutional, Financial interest as Local PI from Adap-timmune, Karyopharm, Lilly.

GGB reports Honoraria from Eli Lilly, Eisai, PharmaMar and MSD; travel grants from PharmaMar, Pfizer and Eli Lilly; advisory board from Eli Lilly and Glaxo Smith Kline.

AB reports Consultancy/Advisory board for Eli Lilly, Eisai, Glaxo Smith Kline; Speakers' fees from PharmaMar; Travel grants from PharmaMar.

JMB reports payment or honoraria for lectures, presentations, speakers bureaus, manuscript writing or educational events from PharmaMar, Lilly, Bayer, Eisai, Daichii; grants or contracts from Pharmamar, Novartis, Eisai, IMMIX Biopharma; payment for expert testimony from Phar-maMar, Lilly, Bayer, Eisai, Daichii; participation on a Data Safety Monitoring Board or Advisory Board for Roche, Lilly, PharmaMar; Leadership or fiduciary role in board, society, committee or advocacy group for Daiichi Sankyo, Karyopharm, Celgene, Pfizer, BMS, Blueprint, Deciphera, Nektar, Forma, Amgen, Lixte, GEIS, CTOS, ESMO, SELNET.

PGC reports honoraria and consultancy or advisory fees from Bayer, Deciphera, Eisai, Eli Lilly, and Pfizer outside the submitted work and institutional financial interest in Advenchen Labora-tories, Amgen Dompe, AROG Pharmaceuticals, Bayer, Blueprint Medicines, Daiichi-Sankyo, De-ciphera, Eisai, Eli Lilly, Epizyme, GlaxoSmithKline, Karyopharm, Novartis, Pfizer, and Pharma-Mar.

Other authors: no competing interests to declare.

Ethical approval: The study was conducted according to the guidelines of the Declaration of Helsinki, and approved by the Ethics Committee of Fondazione IRCCS Istituto Nazionale Tumori di Milano (protocol code $255 / 20$, date $05 / 11 / 2020$ ).

Informed consent: Informed consent was obtained from all subjects involved in the study.

Availability of data and materials: The datasets generated during and/or analysed during the current study are available from the corresponding author on reasonable request.

Acknowledgements: The authors are thankful to our patients who participated in this research to allow us to study a rare disease. 


\section{References}

1. Tan PH, TSe G, Koo JS, Thike AA et al. Fibroepithelial tumours. WHO classification of tumours of the breast (Lyon 2019); 172-176.

2. Zhang Y, Kleer CG. Phyllodes Tumor of the Breast: Histopathologic Features, Differential Diagnosis, and molecular/Genetic Updates. Arch Pathol Lab Med. 2016 Jul;140(7):665-71.

3. Telli ML, Horst KC, Guardino AE, Dirbas FM, Carlson RW. Phyllodes tumors of the breast: natural history, diagnosis, and treatment. J Natl Compr Canc Netw. 2007 Mar;5(3):324-30.

4. Spitaleri G, Toesca A, Botteri E, Bottiglieri L, Rotmensz N, Boselli S, Sangalli C, Catania C, Toffalorio F, Noberasco C, Delmonte A, Luini A, Veronesi P, Colleoni M, Viale G, Zurrida S, Goldhirsch A, Veronesi U, De Pas T. Breast phyllodes tumor: a review of literature and a single center retrospective series analysis. Crit Rev Oncol Hematol. 2013 Nov;88(2):427-36.

5. Neron M, Sajous C, Thezenas S, Piperno-Neumann S, Reyal F, Laé M, Chakiba C, Penel N, Ryckewaert T, Honoré C, Bertucci F, Monneur A, Salas S, Duffaud F, Saada-Bouzid E, Isambert N, Brahmi M, RayCoquard I, Blay JY, Firmin N; French Sarcoma Group (GSF-GETO). Impact of Metastasis Surgery and Alkylating-Agent-Based Chemotherapy on Outcomes of Metastatic Malignant Phyllodes Tumors: A Multicenter Retrospective Study. Ann Surg Oncol. 2020 May;27(5):1693-1699.

6. Mituś JW, Blecharz P, Walasek T, Reinfuss M, Jakubowicz J, Kulpa J. Treatment of Patients with Distant Metastases from Phyllodes Tumor of the Breast. World J Surg. 2016 Feb;40(2):323-8.

7. Ramakant P, Selvamani, Therese MM, Paul MJ. Metastatic Malignant Phyllodes Tumor of the Breast: An Aggressive Disease-Analysis of 7 Cases. Indian J Surg Oncol. 2015 Dec;6(4):363-9.

8. Parkes A, Wang WL, Patel S, Leung CH, Lin H, Conley AP, Somaiah N, Araujo DM, Zarzour M, Livingston JA, Ludwig J, Roland CL, Ravi V, Benjamin RS, Ratan R. Outcomes of systemic therapy in metastatic phyllodes tumor of the breast. Breast Cancer Res Treat. 2021 Apr;186(3):871-882.

9. CTEP.Cancer.gov [homepage on the internet]. Bethesda, MD: NCl; [updated 2017 Nov 27]. Available from:http://ctep.cancer.gov/protocol/Development/electronic_applications/ctc.htm\#ctc_50.

10. Eisenhauer EA, Therasse P, Bogaerts J, Schwartz LH, Sargent D, Ford R, Dancey J, Arbuck S, Gwyther S, Mooney M, Rubinstein L, Shankar L, Dodd L, Kaplan R, Lacombe D, Verweij J. New response evaluation criteria in solid tumours: revised RECIST guideline (version 1.1). Eur J Cancer. 2009 Jan;45(2):228-47.

11. Kaplan, E. L., and Paul Meier. Nonparametric Estimation from Incomplete Observations. Journal of the American Statistical Association, vol. 53, no. 282, 1958, 457-81.

12. Judson I, Verweij J, Gelderblom H, Hartmann JT, Schöffski P, Blay JY, Kerst JM, Sufliarsky J, Whelan J, Hohenberger P, Krarup-Hansen A, Alcindor T, Marreaud S, Litière S, Hermans C, Fisher C, Hogendoorn PC, dei Tos AP, van der Graaf WT; European Organisation and Treatment of Cancer Soft Tissue and Bone Sarcoma Group. Doxorubicin alone versus intensified doxorubicin plus ifosfamide for first-line treatment of advanced or metastatic soft-tissue sarcoma: a randomised controlled phase 3 trial. Lancet Oncol. 2014 Apr;15(4):415-23. 
13. Grignani G, D'Ambrosio L, Pignochino Y, Palmerini E, Zucchetti M, Boccone P, Aliberti S, Stacchiotti S, Bertulli R, Piana R, Miano S, Tolomeo F, Chiabotto G, Sangiolo D, Pisacane A, Dei Tos AP, Novara L, Bartolini A, Marchesi E, D'Incalci M, Bardelli A, Picci P, Ferrari S, Aglietta M. Trabectedin and olaparib in patients with advanced and non-resectable bone and soft-tissue sarcomas (TOMAS): an openlabel, phase 1b study from the Italian Sarcoma Group. Lancet Oncol. 2018 Oct;19(10):1360-1371.

14. Park IH, Kwon Y, Kim EA, Lee KS, Ro J. Major response to sunitinib (Sutene) in metastatic malignant phyllodes tumor of breast. Invest New Drugs. 2009 Aug;27(4):387-8.

15. Casali PG, Abecassis N, Aro HT, Bauer S, Biagini R, Bielack S, Bonvalot S, Boukovinas I, Bovee JVMG, Brodowicz T, Broto JM, Buonadonna A, De Álava E, Dei Tos AP, Del Muro XG, Dileo P, Eriksson M, Fedenko A, Ferraresi V, Ferrari A, Ferrari S, Frezza AM, Gasperoni S, Gelderblom H, Gil T, Grignani G, Gronchi A, Haas RL, Hassan B, Hohenberger P, Issels R, Joensuu H, Jones RL, Judson I, Jutte P, Kaal S, Kasper B, Kopeckova K, Krákorová DA, Le Cesne A, Lugowska I, Merimsky O, Montemurro M, Pantaleo MA, Piana R, Picci P, Piperno-Neumann S, Pousa AL, Reichardt P, Robinson MH, Rutkowski P, Safwat AA, Schöffski P, Sleijfer S, Stacchiotti S, Sundby Hall K, Unk M, Van Coevorden F, van der Graaf WTA, Whelan J, Wardelmann E, Zaikova O, Blay JY; ESMO Guidelines Committee and EURACAN. Soft tissue and visceral sarcomas: ESMO-EURACAN Clinical Practice Guidelines for diagnosis, treatment and follow-up. Ann Oncol. 2018 Oct 1;29(Suppl 4):iv268-iv269.

\section{Figures}


Entire series (56 unique patients): Overall Survival, global

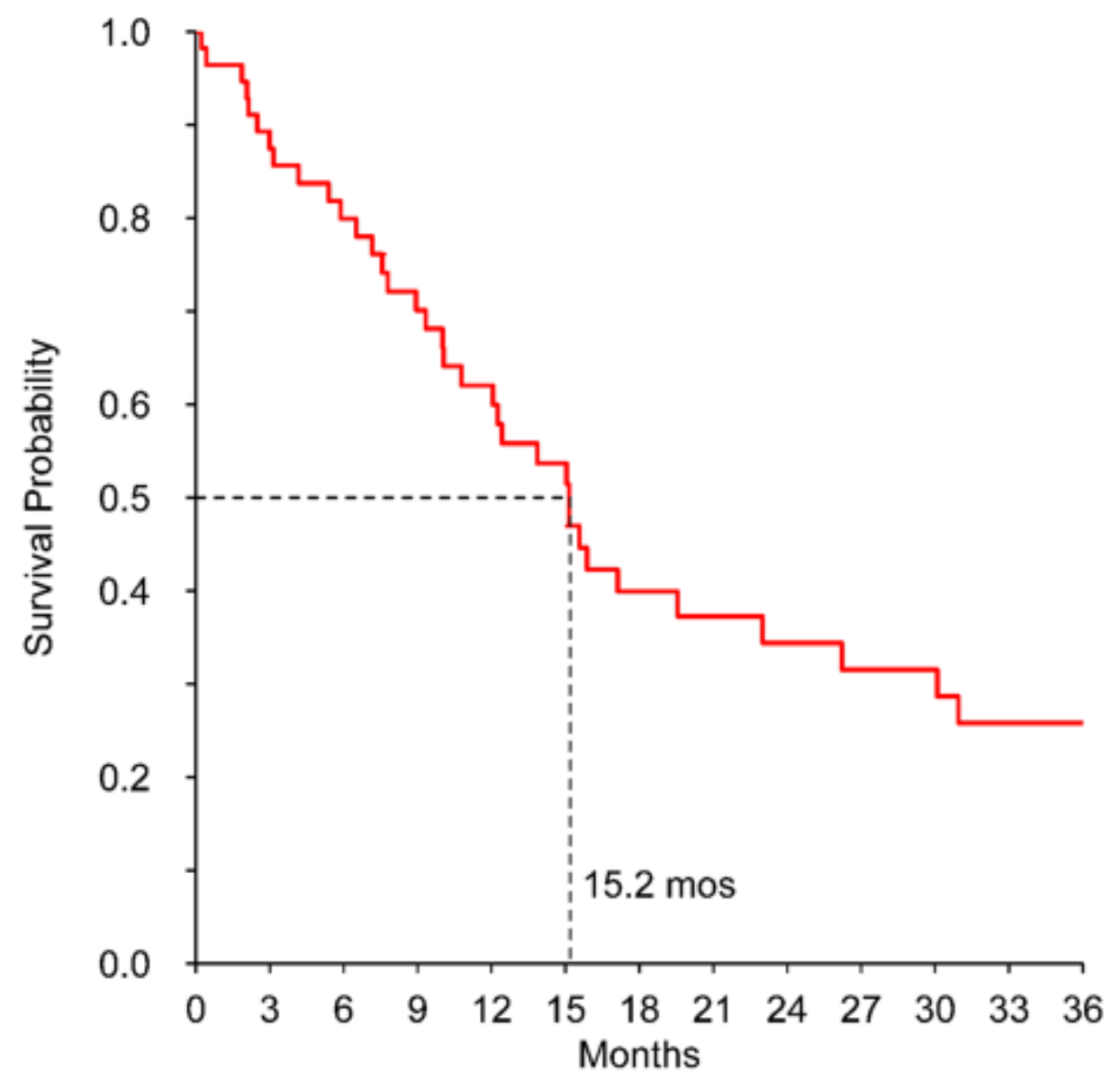

No. pts at risk

$\begin{array}{llllllllllllll}\text { Overall } & 56 & 48 & 42 & 35 & 30 & 24 & 15 & 13 & 12 & 11 & 11 & 8 & 7\end{array}$

Figure 1

OS curve of the entire population in the study 


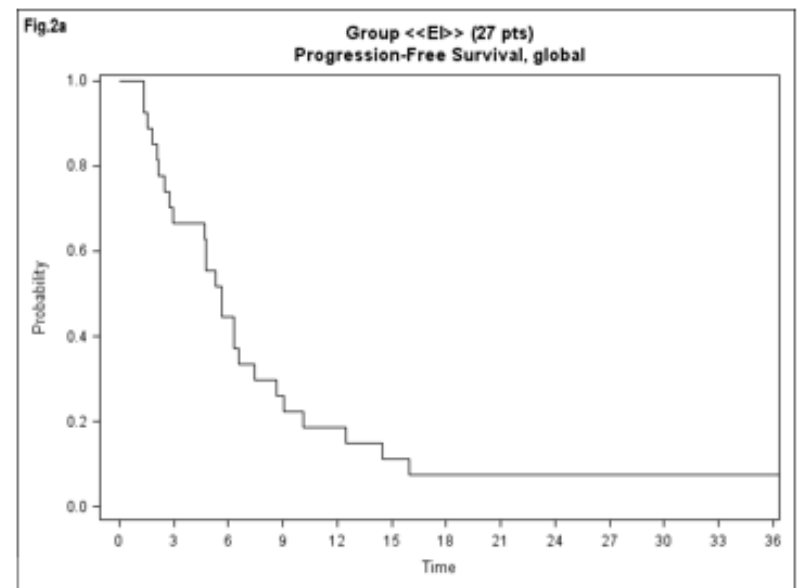

(a)

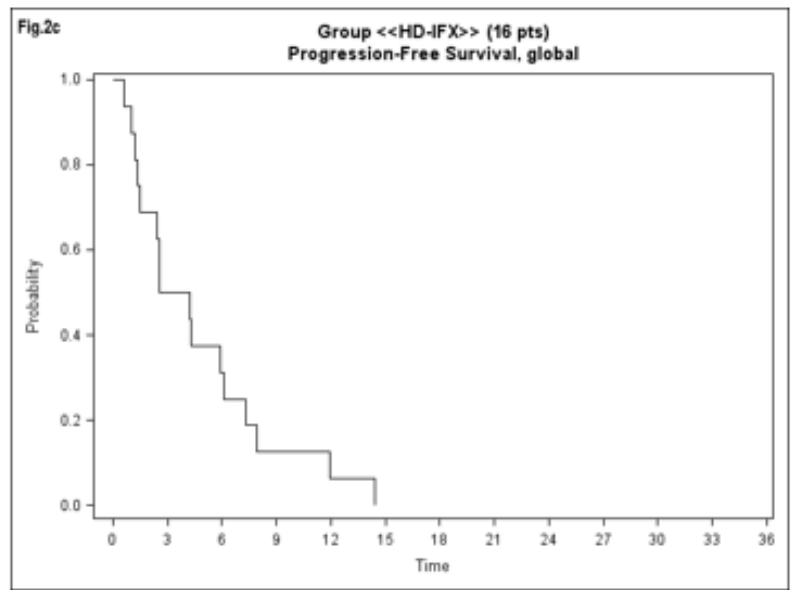

(c)

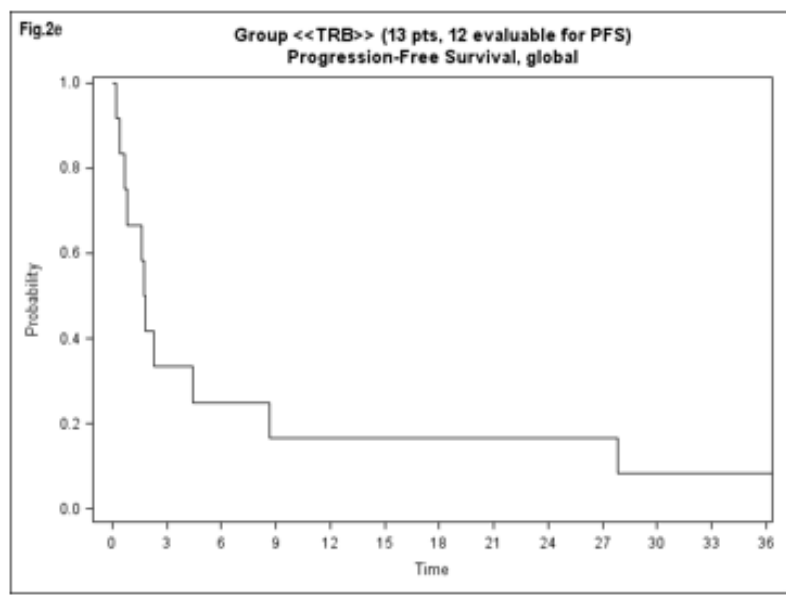

(e)

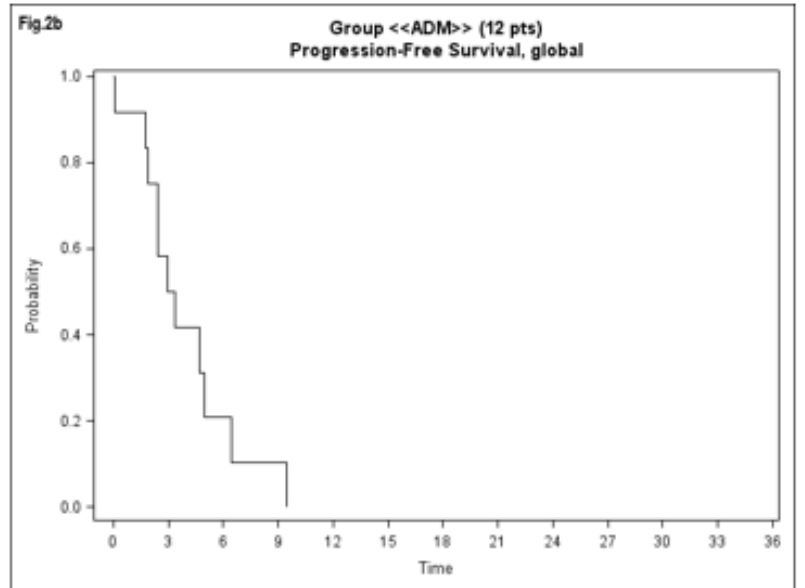

(b)

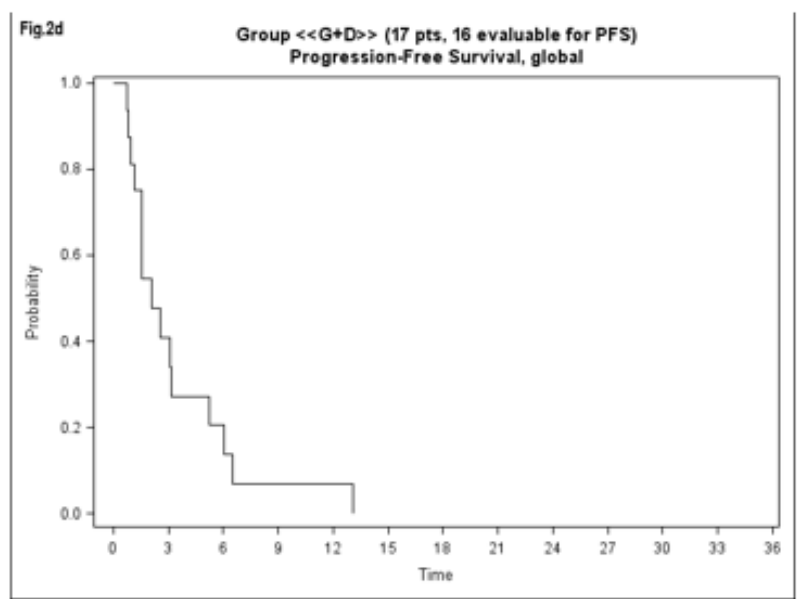

(d)

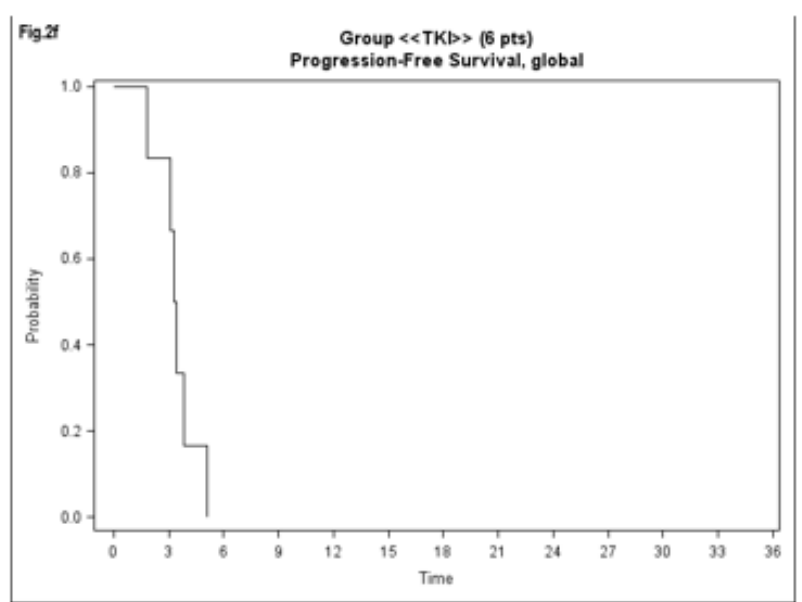

(f)

\section{Figure 2}

(a) PFS curve of pts treated with Al; (b) PFS curve of patients treated with anthracycline; (c) PFS curve of pts treated with HD-IFX; (d) PFS curve of pts treated with gemcitabine-based regimen; (e) PFS curve of pts treated with trabectedin; (f) PFS curve of pts treated with TKI. 